\title{
CHANGE OF INDICES OF THE AMINO ACID COMPOSITION OF RATS' HEARTS AT ARTIFICIAL HYPOBIOSIS
}

\author{
Anna Umanska \\ Postgraduate student \\ Academician M. F. Guliy Department of Biochemistry \\ National University of Life and Environmental Sciences of Ukraine \\ Heroiv Oborony str., 15, Kyiv, Ukraine, 03041 \\ ann.umanska@ukr.net
}

\begin{abstract}
A series of unsolved questions in such sciences as: medicine, veterinary, biology still exist in the modern world. One of them is a search for new promising ways of anaesthetization, at which it would be unnecessary to use apparatuses as an "artificial heart", "artificial ventilation of lungs" at short-term surgical interventions. Just artificial hypobiosis may become one of such methods. Main conditions for creation are a synchronous effect of such factors as hypoxia, hypercapnia, hypothermia. That is why for confirming the safety of this method in pre-clinical studies with a further perspective of using at clinical ones, it is necessary to study the mechanism of an effect and influence of the hypobiotic condition on the homeostasis of the living organism in detail. Rats are the best research object in this case. Just they have a similar physiological structure of such organs as a heart. An urgent question about changes that take place in the amino acid composition under the hypobiotic effect still be unexplained. That is why the aim of the study was to investigate amino acid changes of the rat heart under condition of artificial hypobiosis. White outbred male rats with mass 180-200 g were used in the experiments. The animals were divided in groups: control (intact) and experimental: the condition of artificial hypobiosis (first group) and 24 hours after release from artificial hypobiosis (second group). The number of animals in each group $n=5$. The experiments were conducted according to requirements of "The European convention about protection of vertebral animals, used with experimental or other scientific aims" (Strasbourg, France 1985), by general ethical principles of experiments with animals, approved by the First national congress of Ukraine on bioethics (2001). As a result of the conducted studies, a little decrease of several amino acids under condition of artificial hypobiosis was demonstrated. First of all, a decrease of such amino acids as aspartic, glutamic, isoleucine, leucine, lysine, arginine was observed in rats' hearths under artificial hypobiosis. There was also demonstrated an increase of the level of these amino acids in rats' hearts after 24 hours after release from it.
\end{abstract}

Keywords: hypoxia, hypercapnia, hypotonia, aspartic acid, threonine, serine, isoleucine.

DOI: $10.21303 / 2504-5695.2019 .001073$

\section{Introduction}

A condition of artificial hypobiosis cause a great interest of scientists, because a probability of its model use in medicine, veterinary and animal husbandry as a method of general anaesthetization, conservation of a biological material, therapeutic method and so on is rather high $[1,2]$. A mechanism of biochemical transformations, at which a transfer of organism to the hypometabolic level of life activity takes place, still be unexplained. And also is it possible to use this method safely in clinical practice. Earlier scientists already studied biochemical features of this condition in cardiomyocytes, namely, indices of oxidizing phosphorylation. Analyzing the obtained results, it must be noted, that under condition of artificial hypobiosis, there is observed a partial disintegration of processes of oxidizing phosphorylation in mitochondria of rats' cardiomyocytes at using a substrate - malate and is not observed at using succinate [2]. Based on it, there may be assumed the inhibition of the functional activity of the first conjugating link of oxidation and phosphorylation of the respiratory chain, taking into account that any changes of the functional activity of the last conjugating link were observed. The obtained results is a confirmation of a possibility of endogenic regulation of hypobiosis at the expanse of modifying the functional activity of separate links of the respiratory chain of mitochondria, for example, as at using respiratory chain inhibitors [3]. But changes in the amino acid composition in rats' hearts, taking place in the condition of artificial hypobiosis, are not studied till now. A detail study of 
such indices allows to investigate the mechanism of adaptation to hypobiotic conditions more completely.

The aim of the study is to demonstrate amino acid changes in rats' heart under artificial hypobiosis in the experimental group (condition of artificial hypobiosis and 24 hours after releasing from it) and also to compare amino acid changes in rats' hearts of 3 groups, using different methods of descriptive statistics.

\section{Materials and methods}

White outbred male rats with mass 180-200 g, kept under standard conditions of vivarium, were used in the experiments. They were divided in three groups ( 5 rats in each group): control and experimental (condition of artificial hypobiosis and 24 hours after releasing from it).

For taking the rats in the condition of artificial hypobiosis, the method of BakhmetevGiaja-Andjus was used [4]. The animals were placed in the hermetically closed chamber, volume $3 \mathrm{dm}^{3}$ at temperature $+4{ }^{\circ} \mathrm{C}$. Hypoxia, hypothermia, hypercapnia develop under these factors. In 3-3.5 the decrease of rectal temperature from $37^{\circ} \mathrm{C}$ to $16^{\circ} \mathrm{C}$; decrease of heart rate from 380 to 80 beats per minute were observed; at such conditions animals completely lose mobility, reaction to a pain irritant and reflex to a position that testifies to the artificial hypobiosis development [5].

The experiments were conducted according to requirements of "The European convention about protection of vertebral animals, used with experimental or other scientific aims" (Strasbourg, France 1985), by general ethical principles of experiments with animals, approved by the First national congress of Ukraine on bioethics (2001).

The heart was separated from the rat's body and homogenized with further oxidation at temperature $0{ }^{\circ} \mathrm{C}$ by the mixture of the active acid/phenol. The excess of the oxidizing reagent is disintee grated by sodium disulfite. An oxidized or not oxidized sample (depending on amino acid) is hydrolyzed by saline acid ( $c=6 \mathrm{~mol} / \mathrm{l}$ ) during 23 hours. Hydrolysate is brought to $\mathrm{pH} 2.20$. The content of amino acids in rats' heart was measured according to SSU ISO 13903:2009 «Determination methods of the amino acid content» on the amino acid analyzer AAA4000 Ingos, Czechia [6]. The research results were processed and presented, using the following methods of statistic analysis:

- the method of disruptive statistics;

- the results of the normality verification of data distribution, using Shapiro-Wilk criterion for choosing the statistic criterion for comparing groups;

- the comparison results of the control and experimental group, using Student criterion; criterion;

- the comparison results of the control and experimental group, using Mann-Whitney

- the results of the dynamics estimation in the experimental (hypobiotic) group, using Wilkoxon signed-rank test [7].

\section{Research results}

As a result of the conducted studies, a decrease of such amino acids as aspartic, glutamic, isoleucine, leucine, lysine, arginine was observed in rats' hearths under artificial hypobiosis (Table 1). Other amino acids: threonine, serine, proline, glycine, alanine, valine, tyrosine, phenylalanine, histidine, cystine, methionine still without essential changes.

But a tendency to the renewal of the amino acid level of initial indices (control ones) was observed in experimental group 3 ( 24 hours after releasing from artificial hypobiosis). The results of the normality verification of data distribution are presented in Table 2.

For parameters, which data are distributed normally, a measure of the central tendency is an arithmetic value, and a measure of data dissemination may be a standard deviation. For parameters, which data are distributed not normally, a measure of the central tendency is a median, and a measure of data dissemination may be a minimal and maximal value.

For estimating the substantiality of differences between the groups, there was conducted the one-way ANOVA with further using Tukey HSD test. Realization of the preconditions of the dispersion analysis (normality of residues distribution) was verified using Shapiro-Wilk criterion. The results of multiple comparisons between the groups, using Tukey test, are presented in Table 3. 
Table 1

The comparative analysis of the content of myocardium amino acids in the control and experimental groups of rats, using the methods of descriptive statistics

\begin{tabular}{|c|c|c|c|c|c|c|c|}
\hline Parameter & Group & $\mathbf{n}$ & M & Me & SD & MIN & MAX \\
\hline \multirow{3}{*}{ Aspartic acid } & control & 5 & 2.304 & 2.303 & 0.00301 & 2.3000 & 2.308 \\
\hline & hypobiosis & 5 & 2.128 & 2.128 & 0.00209 & 2.125 & 2.1308 \\
\hline & hypobiosis release & 5 & 2.266 & 2.266 & 0.00060 & 2.2657 & 2.2672 \\
\hline \multirow{3}{*}{ Threonine } & control & 5 & 0.851 & 0.853 & 0.00396 & 0.8438 & 0.8529 \\
\hline & hypobiosis & 5 & 0.827 & 0.826 & 0.00199 & 0.8254 & 0.8301 \\
\hline & hypobiosis release & 5 & 0.840 & 0.840 & 0.00011 & 0.8401 & 0.8404 \\
\hline \multirow{3}{*}{ Serine } & control & 5 & 0.720 & 0.721 & 0.00093 & 0.7187 & 0.7209 \\
\hline & hypobiosis & 5 & 0.705 & 0.706 & 0.00068 & 0.7041 & 0.7058 \\
\hline & hypobiosis release & 5 & 0.716 & 0.717 & 0.00233 & 0.7114 & 0.7167 \\
\hline \multirow{3}{*}{ Glutamic acid } & control & 5 & 2.479 & 2.480 & 0.00162 & 2.4765 & 2.4802 \\
\hline & hypobiosis & 5 & 2.333 & 2.335 & 0.00375 & 2.3264 & 2.335 \\
\hline & hypobiosis release & 5 & 2.407 & 2.403 & 0.00740 & 2.4031 & 2.4198 \\
\hline \multirow{3}{*}{ Proline } & control & 5 & 0.705 & 0.704 & 0.00263 & 0.704 & 0.7101 \\
\hline & hypobiosis & 5 & 0.699 & 0.699 & 0.00060 & 0.6987 & 0.7001 \\
\hline & hypobiosis release & 5 & 0.698 & 0.699 & 0.00075 & 0.6971 & 0.6989 \\
\hline \multirow{3}{*}{ Glycine } & control & 5 & 0.945 & 0.943 & 0.00366 & 0.9429 & 0.9511 \\
\hline & hypobiosis & 5 & 0.931 & 0.930 & 0.00196 & 0.9296 & 0.9341 \\
\hline & hypobiosis release & 5 & 0.936 & 0.936 & 0.00072 & 0.9359 & 0.9375 \\
\hline \multirow{3}{*}{ Alanine } & control & 5 & 1.030 & 1.031 & 0.00193 & 1.0264 & 1.0308 \\
\hline & hypobiosis & 5 & 1.009 & 1.011 & 0.00463 & 1.0009 & 1.0114 \\
\hline & hypobiosis release & 5 & 1.026 & 1.028 & 0.00284 & 1.0212 & 1.0278 \\
\hline \multirow{3}{*}{ Valine } & control & 5 & 0.887 & 0.886 & 0.00171 & 0.8864 & 0.8903 \\
\hline & hypobiosis & 5 & 0.865 & 0.864 & 0.00041 & 0.8642 & 0.8652 \\
\hline & hypobiosis release & 5 & 0.874 & 0.875 & 0.00065 & 0.8732 & 0.8748 \\
\hline \multirow{3}{*}{ Isoleucine } & control & 5 & 0.761 & 0.762 & 0.00314 & 0.7553 & 0.7624 \\
\hline & hypobiosis & 5 & 0.743 & 0.744 & 0.00112 & 0.7412 & 0.7438 \\
\hline & hypobiosis release & 5 & 0.754 & 0.755 & 0.00207 & 0.7506 & 0.7555 \\
\hline \multirow{3}{*}{ Leucine } & control & 5 & 1.516 & 1.517 & 0.00339 & 1.5098 & 1.5176 \\
\hline & hypobiosis & 5 & 1.499 & 1.499 & 0.00046 & 1.4987 & 1.4998 \\
\hline & hypobiosis release & 5 & 1.501 & 1.501 & 0.00037 & 1.5001 & 1.501 \\
\hline \multirow{3}{*}{ Tyrosine } & control & 5 & 1.021 & 1.022 & 0.00171 & 1.0179 & 1.0218 \\
\hline & hypobiosis & 5 & 1.003 & 1.003 & 0.00110 & 1.0007 & 1.0032 \\
\hline & hypobiosis release & 5 & 1.012 & 1.012 & 0.00067 & 1.0109 & 1.0125 \\
\hline \multirow{3}{*}{ Phenylalanine } & control & 5 & 1.388 & 1.387 & 0.00247 & 1.3868 & 1.3924 \\
\hline & hypobiosis & 5 & 1.366 & 1.366 & 0.00053 & 1.3659 & 1.3671 \\
\hline & hypobiosis release & 5 & 1.376 & 1.376 & 0.00022 & 1.3759 & 1.3764 \\
\hline \multirow{3}{*}{ Histidine } & control & 5 & 0.771 & 0.772 & 0.00252 & 0.7663 & 0.772 \\
\hline & hypobiosis & 5 & 0.755 & 0.754 & 0.00243 & 0.7541 & 0.7596 \\
\hline & hypobiosis release & 5 & 0.764 & 0.764 & 0.00186 & 0.7603 & 0.7646 \\
\hline \multirow{3}{*}{ Lysine } & control & 5 & 1.646 & 1.647 & 0.00425 & 1.6379 & 1.6474 \\
\hline & hypobiosis & 5 & 1.625 & 1.624 & 0.00150 & 1.6239 & 1.6273 \\
\hline & hypobiosis release & 5 & 1.634 & 1.635 & 0.00083 & 1.6328 & 1.6348 \\
\hline \multirow{3}{*}{ Arginine } & control & 5 & 1.105 & 1.103 & 0.00443 & 1.1029 & 1.1128 \\
\hline & hypobiosis & 5 & 1.066 & 1.065 & 0.00083 & 1.0653 & 1.0672 \\
\hline & hypobiosis release & 5 & 1.096 & 1.098 & 0.00355 & 1.0898 & 1.0979 \\
\hline \multirow{3}{*}{ Cystine } & control & 5 & 0.299 & 0.298 & 0.00147 & 0.2984 & 0.3017 \\
\hline & hypobiosis & 5 & 0.281 & 0.280 & 0.00233 & 0.2801 & 0.2854 \\
\hline & hypobiosis release & 5 & 0.294 & 0.292 & 0.00331 & 0.2924 & 0.2998 \\
\hline \multirow{3}{*}{ Methionine } & control & 5 & 0.515 & 0.513 & 0.00387 & 0.5134 & 0.5221 \\
\hline & hypobiosis & 5 & 0.501 & 0.501 & 0.00035 & 0.5005 & 0.5014 \\
\hline & hypobiosis release & 5 & 0.512 & 0.512 & 0.00092 & 0.5101 & 0.5123 \\
\hline
\end{tabular}

Note: $n$-mean ariphmetuical, $M-m c g / g$ of amino acid, Me - median, SD - standard deviation, MIN-minimum, MAX-maximum 
Table 2

The normality verification of data distribution of the experimental and control groups of the rats' myocardium amino acid composition using Shapiro-Wilk criterion

\begin{tabular}{|c|c|c|c|c|c|}
\hline Parameter & Group & Shapiro-Wilk statistics & df & $\mathbf{p}$ & Conclusion about normality* \\
\hline \multirow{3}{*}{ Aspartic acid } & control & 0.966 & 5 & 0.848 & Normal \\
\hline & hypobiosis & 0.965 & 5 & 0.841 & normal \\
\hline & hypobiosis release & 0.788 & 5 & 0.064 & normal \\
\hline \multirow{3}{*}{ Threonine } & control & 0.588 & 5 & 0.000 & Not normal \\
\hline & hypobiosis & 0.716 & 5 & 0.014 & normal \\
\hline & hypobiosis release & 0.961 & 5 & 0.814 & normal \\
\hline \multirow{3}{*}{ Serine } & control & 0.636 & 5 & 0.002 & Not normal \\
\hline & hypobiosis & 0.759 & 5 & 0.036 & normal \\
\hline & hypobiosis release & 0.581 & 5 & 0.000 & Not normal \\
\hline \multirow{3}{*}{ Glutamic acid } & control & 0.575 & 5 & 0.000 & Not normal \\
\hline & hypobiosis & 0.582 & 5 & 0.000 & Not normal \\
\hline & hypobiosis release & 0.562 & 5 & 0.000 & Not normal \\
\hline \multirow{3}{*}{ Proline } & control & 0.669 & 5 & 0.004 & Not normal \\
\hline & hypobiosis & 0.669 & 5 & 0.004 & Not normal \\
\hline & hypobiosis release & 0.656 & 5 & 0.003 & Not normal \\
\hline \multirow{3}{*}{ Glycine } & control & 0.560 & 5 & 0.000 & Not normal \\
\hline & hypobiosis & 0.610 & 5 & 0.001 & Not normal \\
\hline & hypobiosis release & 0.552 & 5 & 0.000 & Not normal \\
\hline \multirow{3}{*}{ Alanine } & control & 0.571 & 5 & 0.000 & Not normal \\
\hline & hypobiosis & 0.580 & 5 & 0.000 & Not normal \\
\hline & hypobiosis release & 0.601 & 5 & 0.001 & Not normal \\
\hline \multirow{3}{*}{ Valine } & control & 0.619 & 5 & 0.001 & Not normal \\
\hline & hypobiosis & 0.799 & 5 & 0.079 & normal \\
\hline & hypobiosis release & 0.661 & 5 & 0.004 & Not normal \\
\hline \multirow{3}{*}{ Isoleucine } & control & 0.564 & 5 & 0.000 & Not normal \\
\hline & hypobiosis & 0.626 & 5 & 0.001 & Not normal \\
\hline & hypobiosis release & 0.625 & 5 & 0.001 & Not normal \\
\hline \multirow{3}{*}{ Leucine } & control & 0.585 & 5 & 0.000 & Not normal \\
\hline & hypobiosis & 0.723 & 5 & 0.016 & normal \\
\hline & hypobiosis release & 0.648 & 5 & 0.003 & Not normal \\
\hline \multirow{3}{*}{ Tyrosine } & control & 0.574 & 5 & 0.000 & Not normal \\
\hline & hypobiosis & 0.591 & 5 & 0.000 & Not normal \\
\hline & hypobiosis release & 0.650 & 5 & 0.003 & Not normal \\
\hline \multirow{3}{*}{ Phenylalanine } & control & 0.567 & 5 & 0.000 & Not normal \\
\hline & hypobiosis & 0.607 & 5 & 0.001 & Not normal \\
\hline & hypobiosis release & 0.552 & 5 & 0.000 & Not normal \\
\hline \multirow{3}{*}{ Histidine } & control & 0.567 & 5 & 0.000 & Not normal \\
\hline & hypobiosis & 0.582 & 5 & 0.000 & Not normal \\
\hline & hypobiosis release & 0.611 & 5 & 0.001 & Not normal \\
\hline \multirow{3}{*}{ Lysine } & control & 0.552 & 5 & 0.000 & Not normal \\
\hline & hypobiosis & 0.580 & 5 & 0.000 & Not normal \\
\hline & hypobiosis release & 0.638 & 5 & 0.002 & Not normal \\
\hline \multirow{3}{*}{ Arginine } & control & 0.552 & 5 & 0.000 & Not normal \\
\hline & hypobiosis & 0.653 & 5 & 0.003 & Not normal \\
\hline & hypobiosis release & 0.586 & 5 & 0.000 & Not normal \\
\hline \multirow{3}{*}{ Cystine } & control & 0.573 & 5 & 0.000 & Not normal \\
\hline & hypobiosis & 0.588 & 5 & 0.000 & Not normal \\
\hline & hypobiosis release & 0.552 & 5 & 0.000 & Not normal \\
\hline \multirow{3}{*}{ Methionine } & control & 0.568 & 5 & 0.000 & Not normal \\
\hline & hypobiosis & 0.745 & 5 & 0.027 & Normal \\
\hline & hypobiosis release & 0.630 & 5 & 0.002 & Not normal \\
\hline
\end{tabular}

Note: $d f$-number of animals in groups, $p$-significance level of the criterion 
Table 3

The comparison of the control and experimental groups of the amino acid composition of the rats' myocardium. using Tukey HSD test

\begin{tabular}{|c|c|c|c|c|c|}
\hline Parameter & (I) Group & (J) Group & Difference of averages (I-J) & Std error & p-value \\
\hline \multirow{3}{*}{ Rank for «Aspartic acid» } & \multirow{2}{*}{ Control } & Hypobiosis & 0.176 & 0.001 & 0.000 \\
\hline & & Hypobiosis release & 0.037 & 0.001 & 0.000 \\
\hline & hypobiosis & Hypobiosis release & -0.138 & 0.001 & 0.000 \\
\hline \multirow{3}{*}{ Rank for «Threonine» } & \multirow{2}{*}{ Control } & Hypobiosis & 10.000 & 0.975 & 0.000 \\
\hline & & Hypobiosis release & 5.000 & 0.975 & 0.001 \\
\hline & hypobiosis & Hypobiosis release & -5.000 & 0.975 & 0.001 \\
\hline \multirow{3}{*}{ Ranks for «Serine» } & \multirow{2}{*}{ Control } & Hypobiosis & 10.000 & 0.983 & 0.000 \\
\hline & & Hypobiosis release & 5.000 & 0.983 & 0.001 \\
\hline & hypobiosis & Hypobioss release & -5.000 & 0.983 & 0.001 \\
\hline \multirow{3}{*}{ Ranks for «Glutamic acid» } & \multirow{2}{*}{ Control } & Hypobiosis & 10.000 & 0.894 & 0.000 \\
\hline & & Hypobiosis release & 5.000 & 0.894 & 0.000 \\
\hline & hypobiosis & Hypobioss release & -5.000 & 0.894 & 0.000 \\
\hline \multirow{3}{*}{ Ranks for «Proline» } & \multirow{2}{*}{ Control } & Hypobiosis & 6.600 & 1.611 & 0.004 \\
\hline & & Hypobiosis release & 8.400 & 1.611 & 0.001 \\
\hline & hypobiosis & Hypobioss release & 1.800 & 1.611 & 0.522 \\
\hline \multirow{3}{*}{ Ranks for «Glycine» } & \multirow{2}{*}{ Control } & Hypobiosis & 10.000 & 0.866 & 0.000 \\
\hline & & Hypobiosis release & 5.000 & 0.866 & 0.000 \\
\hline & hypobiosis & Hypobioss release & -5.000 & 0.866 & 0.000 \\
\hline \multirow{3}{*}{ Ranks for «Alanine» } & \multirow{2}{*}{ Control } & Hypobiosis & 9.200 & 1.415 & 0.000 \\
\hline & & Hypobiosis release & 3.400 & 1.415 & 0.079 \\
\hline & hypobiosis & Hypobioss release & -5.800 & 1.415 & 0.004 \\
\hline \multirow{3}{*}{ Ranks for «Valine» } & \multirow{2}{*}{ Control } & Hypobiosis & 10.000 & 0.922 & 0.000 \\
\hline & & Hypobiosis release & 5.000 & 0.922 & 0.000 \\
\hline & hypobiosis & Hypobioss release & -5.000 & 0.922 & 0.000 \\
\hline \multirow{3}{*}{ Ranks for «Isoleucine» } & \multirow{2}{*}{ Control } & Hypobiosis & 9.800 & 1.097 & 0.000 \\
\hline & & Hypobiosis release & 4.600 & 1.097 & 0.003 \\
\hline & hypobiosis & Hypobioss release & -5.200 & 1.097 & 0.001 \\
\hline \multirow{3}{*}{ Ranks for «Leucine» } & \multirow{2}{*}{ Control } & Hypobiosis & 10.000 & 0.913 & 0.000 \\
\hline & & Hypobiosis release & 5.000 & 0.913 & 0.000 \\
\hline & hypobiosis & Hypobioss release & -5.000 & 0.913 & 0.000 \\
\hline & Control & Hypobiosis & 10.000 & 0.940 & 0.000 \\
\hline Ranks for «Tyrosine» & Comtior & Hypobiosis release & 5.000 & 0.940 & 0.000 \\
\hline & hypobiosis & Hypobioss release & -5.000 & 0.940 & 0.000 \\
\hline & Control & Hypobiosis & 10.000 & 0.837 & 0.000 \\
\hline Ranks for «Phenylalanine» & 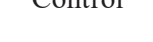 & Hypobiosis release & 5.000 & 0.837 & 0.000 \\
\hline & hypobiosis & Hypobioss release & -5.000 & 0.837 & 0.000 \\
\hline & Control & Hypobiosis & 0.016 & 0.001 & 0.000 \\
\hline Ranks for Histidine & Control & Hypobiosis release & 0.007 & 0.001 & 0.001 \\
\hline & hypobiosis & Hypobioss release & -0.008 & 0.001 & 0.000 \\
\hline & Control & Hypobiosis & 10.000 & 0.856 & 0.000 \\
\hline Ranks for «Lysine» & contror & Hypobiosis release & 5.000 & 0.856 & 0.000 \\
\hline & hypobiosis & Hypobioss release & -5.000 & 0.856 & 0.000 \\
\hline & Control & Hypobiosis & 0.039 & 0.002 & 0.000 \\
\hline Ranks for Arginine & Control & Hypobiosis release & 0.009 & 0.002 & 0.003 \\
\hline & hypobiosis & Hypobioss release & -0.030 & 0.002 & 0.000 \\
\hline & Control & Hypobiosis & 9.200 & 1.361 & 0.000 \\
\hline Ranks for «Cystine» & comtror & Hypobiosis release & 3.400 & 1.361 & 0.067 \\
\hline & hypobiosis & Hypobioss release & -5.800 & 1.361 & 0.003 \\
\hline & Control & Hypobiosis & 10.000 & 0.894 & 0.000 \\
\hline Ranks for «Methionine» & Comtior & Hypobiosis release & 5.000 & 0.894 & 0.000 \\
\hline & hypobiosis & Hypobioss release & -5.000 & 0.894 & 0.000 \\
\hline
\end{tabular}

Note: $p$-significance level of the criterion 


\section{Discussion of the research results}

The decrease of aspartic acid in rats' hearts at artificial hypobiosis is explained by the fact that the activity of the endocrine and nervous systems decreases, because it acts as a neuromediator in CNS [8].

Glutamic acid functions as a transport of ammonia in the organism, and also is a neuromediate amino acid, so its decrease confirms the hypothesis that centers of pain feeling inactivate at artificial hypobiosis. The isoleucine decrease in the cardiac muscle is probably explained by the mobile activity decrease, because this amino acid is an energy supplier to muscles [9]. The leucine level decrease in the rat's myocardium at artificial hypobiosis testifies that it can be spent for supporting an energetic function at the cellular level, because this amino acid actively participates in reactions of oxidizing decarboxidation of 3-keto acids [10]. The arginine decrease is explained by decreasing nervous impulses conduction at artificial hypobiosis that give a ground to assume that at the effect of hypobiotic factors, surgical interventions are possible, because pain feelings are absent [11].

Based on the analytic results from Table 3, it may be concluded, that all groups by all parameters of the amino acid composition of the rats' heart were statistically essentially different. Using the well-known statistical methods, it has been established, that all amino acids were statistically essentially different from the control group in the rats' myocardium at artificial hypobiosis [12].

\section{Conclusions}

The research demonstrated the increase of the following amino acids in the rats' heart at artificial hypobiosis: aspartic, glutamic, isoleucine, leucine, lysine and arganine. But there was demonstrated the increase of the level of these amino acids in experimental group 3 (24 after releasing from hypobiotic factors).

According to the results of the multiple comparisons between the groups (control, experimental 1 and experimental 2) using Tukey test, it has been established, that all parameters of amino acids are different.

As a result of the conducted studies, a cognitive conclusion that hypobiosis as a method of general anaesthetization may be used in medical and veterinary practices after further studies, may be made.

\section{References}

[1] Banerjee, P. S., Ma, J., Hart, G. W. (2015). Diabetes-associated dysregulation ofO-GlcNAcylation in rat cardiac mitochondria. Proceedings of the National Academy of Sciences, 112 (19), 6050-6055. doi: https://doi.org/10.1073/pnas. 1424017112

[2] Morozova, V. S. (2013). Functioning of the antioxidant system of rat myocardium for condition of artificial hypobyosis. Bulletin of Biology and Medicine, 3 (2), 103.

[3] Melnychuk, D. O., Melnychuk, S. D., Arnauta, O. V. (2004). Influence of carbon dioxide on the environment preservation of red blood cells in stored blood of animals. Scientific Bulletin of NAU, 75, 163-165.

[4] Melnychuk, S. D. (2001). Key figures acid-base status blood and metabolic hibernation and when general anesthesia for the amputation. Ukrainian Biochemical Journal, 73 (6), 80-83.

[5] Melnychuk, S. D., Melnychuk, D. O. (2007). Animal dormancy (molecular mechanisms and practical for Agriculture and Medicine). Kyiv: Publishing Center NAU, 220.

[6] Sorochan, O. O., Shtemenko, N. I. (2005). Methods of analysis of amino acids. Kyiv.

[7] Glanz, S. (1999). Medico-biological statistics. Moscow: Practice, 460.

[8] Kolomiytseva, I. K. (2011). Lipids in mammalian hibernation and artificial hypobiosis. Biochemistry (Moscow), 76 (12), 1291-1299. doi: https://doi.org/10.1134/s0006297911120029

[9] Dahl, R. H., Berg, R. M. G., Taudorf, S., Bailey, D. M., Lundby, C., Christensen, M. et. al. (2019). Transcerebral exchange kinetics of large neutral amino acids during acute inspiratory hypoxia in humans. Scandinavian Journal of Clinical and Laboratory Investigation, 79 (8), 595-600. doi: https://doi.org/10.1080/00365513.2019.1683762 
[10] Logvinovich, O. S., Aksenova, G. E. (2013). Ornithine decarboxylase in mammalian organs and tissues at hibernation and artificial hypobiosis. Zh Obshch Biol, 74 (3), 180-189.

[11] De Oliveira, R. W., Julian, G. S., Perry, J. C., Tufik, S., Chagas, J. R. (2018). Chronic intermittent hypoxia induces changes on the expression and activity of neprilysin (EC 3.4.24.11) in the brain of rats. Neuroscience Letters, 678, 43-47. doi: https://doi.org/ 10.1016/j.neulet.2018.04.045

[12] Jastroch, M., Giroud, S., Barrett, P., Geiser, F., Heldmaier, G., Herwig, A. (2016). Seasonal Control of Mammalian Energy Balance: Recent Advances in the Understanding of Daily Torpor and Hibernation. Journal of Neuroendocrinology, 28 (11). doi: https://doi.org/10.1111/jne.12437 